\title{
Trend of Respiratory Tract-Associated Streptococcus dysgalactiae Subsp. Equisimilius at General Japanese Hospital from 2013 to 2015
}

\author{
Masaaki Minami ${ }^{*}$, Ryoko Sakakibara², Taichi Imura², Mika Watanabe ${ }^{2}$, Hideo Morita², \\ Naoto Kanemaki ${ }^{3}$, Michio Ohta ${ }^{4}$ \\ ${ }^{1}$ Department of Bacteriology, Graduate School of Medical Sciences, Nagoya City University, Nagoya, Japan \\ ${ }^{2}$ Department of Clinical Investigation, Daido Hospital, Nagoya, Japan \\ ${ }^{3}$ Department of Gastroenterology, Daido Hospital, Nagoya, Japan \\ ${ }^{4}$ School of Nursing, Sugiyama Jyogakuen University, Nagoya, Japan \\ Email:^minami@med.nagoya-cu.ac.jp
}

How to cite this paper: Minami, M., Sakakibara, R., Imura, T., Watanabe, M., Morita, H., Kanemaki, N. and Ohta, M. (2016) Trend of Respiratory Tract-Associated Streptococcus dysgalactiae Subsp. Equisimilius at General Japanese Hospital from 2013 to 2015. Journal of Biosciences and Medicines, 4, 11 17.

http://dx.doi.org/10.4236/jbm.2016.412002

Received: September 19, 2016 Accepted: November 24, 2016 Published: December 1, 2016

\section{Abstract}

Streptococcus dysgalactiae subsp. equisimilius are pathogenic bacteria which cause various infectious diseases from acute pharyngitis to streptococcal toxic shock syndrome. Although Streptococcus dysgalactiae subsp. equisimilius infection has been increasing recently, the recent characteristic investigation of respiratory tract associated Streptococcus dysgalactiae subsp. equisimilius has not been performed in Japan. In this study, we analyzed the relationship between respiratory tract and norespiratory tract associated Streptococcus dysgalactiae subsp. equisimilius by investigating the recent clinical characteristics and antimicrobial susceptible patterns of 98 Streptococcus dysgalactiae subsp. equisimilius isolated during 2013-2015 in Japan. There was significant difference of gender between respiratory tract and no respiratory tract associated Streptococcus dysgalactiae subsp. equisimilius. No Streptococcus dysgalactiae subsp. equisimilius from no respiratory tract were found under 18 years-old patients. The prevalence of clindamycin non susceptible Streptococcus dysgalactiae subsp. equisimilius isolates from respiratory tract was significant greater than those from non-respiratory tract. We also analyzed the relationship between respiratory tract associated Streptococcus dysgalactiae subsp. equisimilius and Streptococcus pyogenes in 2014.There was no significant difference of gender between Streptococcus dysgalactiae subsp. equisimilius and Streptococcus pyogenes. The numbers of Streptococcus pyogenes-infected patients were significantly greater than those of Streptococcus dysgalactiae subsp. equisimilius-infected patients in children from 1 to 18 years old. From antimicrobial resistance, we found that the numbers of clarithromycin resistant Streptococcus pyogenes were greater than those 
of clarithromycin resistant Streptococcus dysgalactiae subsp. equisimilius among respiratory tract isolation. Our results suggest that the clinical and antimicrobial susceptible patterns are useful for screening the respiratory tract associated Streptococcus dysgalactiae subsp. equisimilius infection.

\section{Keywords}

Streptococcus dysgalactiaesusp. equsimilius, Susceptibility, Antimicrobial Resistance, Epidemiology

\section{Introduction}

Most $\beta$-hemolytic streptococcus from human are categolized as Streptococcus pyogenes, Streptococcus agalactiae, and Streptococcus dysgalactiae subsp. Equisimilis [1]. In contrast to Streptococcus pyogenes and Streptococcus agalactiae, Streptococcus dysgalactiae subsp. equisimilis had been long considered as commensal organisms that have low virulence as opportunistic pathogens [2]. In 1996, Streptococcus dysgalactiae subsp. Equisimilis was proposed to be a new streptococcal taxon [3]. It has also been reported to cause a wide variety of human infections such as pharyngitis, cellulitis, sepsis, meningitis, endocarditis, and streptococcal toxic shock syndrome (STSS) [4].

Streptococcus dysgalactiae subsp. equisimilis have many virulence factors shared with Streptococcus pyogenes, such as M protein, streptolysin O, streptolysin S, streptokinase, and streptococcal inhibitory of complement lysis [5] [6]. Although Streptococcus dysgalactiae subsp. equisimilis infection has been increasing recently [7] [8], the recent characteristic investigation of respiratory tract associated Streptococcus dysgalactiae subsp. equisimilis has not been performed in Japan [9] [10]. The present study was conducted to find out the relationship between respiratory tract and no respiratory tract associated Streptococcus dysgalactiae subsp. equisimilis by investigating the recent clinical characteristics and antimicrobial susceptible patterns of Streptococcus dysgalactiae subsp. equisimilis isolated in Japan.

\section{Materials and Methods}

\subsection{Strains and Clinical Data Collection}

A total of 98 Streptococcus dysgalactiae subsp. equisimilius from 2013 to 2015 and 161 Streptococcus pyogenes from 2014 was obtained at Daido Hospital. Daido Hospital is a 404-bed private general hospital in the central region of Japan. We used medical records appended to clinical species for the analysis of clinical feature at Daido Hospital. We considered several isolates from the same region of the same patient as one isolate per one patient for the analysis in this study. All streptococcus isolates were identified by standard conventional biochemical methods or the VITEK2 system (bioMérieux, Durham NC, USA). Our experimental design was approved by the ethics committee at Daido hospital. 


\subsection{Antimicrobial Susceptibility Analysis}

Streptococcus dysgalactiae subsp. equisimilius and Streptococcus pyogenes isolates were examined for 15 antibiotic susceptibilities as follows sulbactam/ampicillin, piperacillin, amoxicillin, cefotiam, flomoxef, ceftazidime, ceftriaxone, vancomycin, fosfomycin, panipenem, meropenem, clarithromycin, clindamycin, minocycline, ciprofloxacin. Minimal inhibitory concentrations (MICs) were determined at clinical laboratory in Daido Hospital using broth micro dilution methodology with the VITEK2 system. Evaluation of susceptibilities was calculated based on Clinical Laboratory Standard Institute (CLSI) break point [11].

\subsection{Statistical Analysis of the Data}

We conducted the statistical analysis with the chi-squared test or Fisher's exact test when appropriate. Differences were considered significant when $p$ was $<0.05$.

\section{Results}

First of all, we confirmed that all Streptococcus dysgalactiae subsp. equisimilis and Streptococcus pyogenes isolates had beta hemolytic activity in this study. Next, we compared the differences between respiratory tract and no respiratory tract isolates in this study. Respiratory tract isolates were defined as isolation from pharynx, nasal discharge, tonsillar, and sputum. After that, we compared the differences of clinical and bacteriological features between respiratory tract and no respiratory tract isolations in this study. Firstly, we analyzed the relationship between respiratory tract and no respiratory tract related Streptococcus dysgalactiae subsp. equisimilis. Total ninety-eight were isolated among which 53 were from respiratory tract and 45 were from non-respiratory tract. Table 1 demonstrated the comparative analysis of Streptococcus dysgalactiae subsp. equisimilis between respiratory tract and no respiratory tract. Ninety-eight Streptococcus dysgalactiae subsp. equisimilis were isolated among which 36 were from male and 17 were from female. The numbers of males in respiratory tract disease were significantly greater than those of males in no respiratory tract disease $(p=$ 0.04). The age incidences among $0-1$ and among $1-18$ years age group were 11 (11.2\%) and 17 (17.3\%), respectively. These were only isolated from respiratory tract disease. The age incidence among 19 - 64 years age group, 17(17.3\%) [respiratory tract-4, no respiratory tract-13], and in over 65-years, it was 53 (54.1\%) [respiratory tract-21, no respiratory tract-32]. Furthermore the numbers of aged-patient were significantly increasing gradually both respiratory and no respiratory disease $(p=0.04)$. The results of antimicrobial susceptible patterns of Streptococcus dysgalactiae subsp. equisimilis isolates against four antibiotics were shown in Table 1. Because other antibiotics were the most active antibiotics with $100 \%$ susceptible rates against Streptococcus dysgalactiae subsp. equisimilis and Streptococcus pyogenes. Although there were no significant differences of three antimicrobial (clarithromycin, minocycline, ciprofloxacin) susceptibility between respiratory tract and no respiratory tract Streptococcus dysgalactiae subsp. equisimilis, the prevalence of clindamycin resistant from respiratory 
tract was significant greater than that from no respiratory tract $(p=0.02)$. We next analyzed the relationship between respiratory tract associated Streptococcus dysgalactiae subsp. equisimilis and Streptococcus pyogenes. Because Streptococcus pyogenes are more popular than related Streptococcus dysgalactiae subsp. equisimilis in terms of respiratory tract pathogen. Table 2 demonstrated the comparative analysis between Streptococcus dysgalactiae subsp. equisimilis isolates and Streptococcus pyogenes from respiratory tract. There was no significant difference of gender between Streptococcus dysgalactiae subsp. equisimilis and Streptococcus pyogenes. Both bacteria were isolated

Table 1. Clinical characteristics between respiratory tract and non-respiratory tract Streptococcus dysgalactiae subsp. equisimilis.

\begin{tabular}{|c|c|c|c|c|c|c|c|}
\hline & & \multicolumn{3}{|c|}{ Respiratoty tract } & \multicolumn{3}{|c|}{ Norespiratory tract } \\
\hline & & $\begin{array}{c}2013 \\
(n=29)\end{array}$ & $\begin{array}{c}2014 \\
(\mathrm{n}=14)\end{array}$ & $\begin{array}{c}2015 \\
(\mathrm{n}=10)\end{array}$ & $\begin{array}{c}2013 \\
(\mathrm{n}=14)\end{array}$ & $\begin{array}{c}2014 \\
(\mathrm{n}=21)\end{array}$ & $\begin{array}{c}2015 \\
(n=10)\end{array}$ \\
\hline \multirow[t]{2}{*}{ Gender } & Male & 17 & 10 & 9 & 4 & 14 & 3 \\
\hline & Female & 12 & 4 & 1 & 10 & 7 & 7 \\
\hline \multirow[t]{4}{*}{ Age } & $0-1$ & 7 & 3 & 1 & 0 & 0 & 0 \\
\hline & $1-18$ & 11 & 2 & 4 & 0 & 0 & 0 \\
\hline & $19-64$ & 2 & 2 & 0 & 2 & 5 & 6 \\
\hline & $65-$ & 9 & 7 & 5 & 12 & 16 & 4 \\
\hline \multirow[t]{4}{*}{ No-susceptibility } & Clarithromycin & 8 & 6 & 3 & 2 & 6 & 2 \\
\hline & Clindamycin & 8 & 4 & 2 & 1 & 1 & 1 \\
\hline & Ciprofloxacin & 10 & 4 & 1 & 7 & 9 & 1 \\
\hline & Monocycline & 3 & 4 & 3 & 1 & 3 & 3 \\
\hline
\end{tabular}

Table 2. Clinical characteristics between respiratory tract Streptococcus dysgalactiae subsp. equisimilis and respiratory tract Streptococcus pyogenes.

\begin{tabular}{cccc}
\hline \multirow{2}{*}{ Gender } & Male & $\begin{array}{c}\text { Streptococcus } \\
\text { pyogenes }(\%)\end{array}$ & $\begin{array}{c}\text { Streptococcus dysgalactiae } \\
\text { subsp. equisimilius (\%) }\end{array}$ \\
\hline \multirow{2}{*}{ Age } & Female & 66.2 & 71.4 \\
& $0-1$ & 33.8 & 28.6 \\
& $1-18$ & 4.4 & 21.4 \\
No-susceptibility & $19-64$ & 3.7 & 14.3 \\
& $65-$ & 2.2 & 14.3 \\
& Clarithromycin & 66.9 & 50 \\
& Clindamycin & 27.9 & 42.9 \\
& Ciprofloxacin & 33.8 & 28.6 \\
& Mnocycline & 18.4 & 28.6 \\
\hline
\end{tabular}


from more male than female. The numbers of Streptococcus pyogenes-infected patients were significantly greater than those of Streptococcus dysgalactiae subsp. equisimilisinfected patients in children from 1 to 18 years old $(p<0.01)$. Clarithromycin was less antimicrobial effective in respiratory tract disease than in no respiratory tract disease significantly $(p<0.01)$. Because the numbers of Streptococcus pyogenes were also significantly greater than those of Streptococcus dysgalactiae subsp. equisimilis according to clarithromycin-resistance. However, the numbers of Streptococcus dysgalactiae subsp. equisimilis tended to be greater than those of Streptococcus pyogenes according to minocycline-resistance. There were no significant differences of other three antibiotics between respiratory tract and no respiratory tract isolation.

\section{Discussion}

In this study, we described the characteristics of respiratory tract associated Streptococcus dysgalactiae subsp. equisimilis isolates at general hospital in the central of Japan from 2013 to 2015. We found the numbers of bacteria from respiratory tract were about 1.2 times as same as those from no respiratory tract. In the analysis of gender, we did notfind any significant differences of gender in our previous report [8]. However, the numbers of Streptococcus dysgalactiae subsp. equisimilis isolated from male were greater than those from femalein current study. We also clarified Streptococcus dysgalactiae subsp. equisimilis with age distribution. The previous our study showed that the numbers of Streptococcus dysgalactiae subsp. equisimilis isolated from children (1 to 10 year-old) and elder people (over 65 year-old) were most popular. However, the numbers of no respiratory tract associated Streptococcus dysgalactiae subsp. equisimilis isolated from elder people (over 65 year-old) were most popular [8]. In the analysis of antimicrobial susceptibility, previous our study demonstrated that the prevalence of clarithromycin resistant, clindamycin resistant, minocycline-resistant, and ciprofloxacin-resistant Streptococcus dysgalactiae subsp. equisimilis were 37\%, 23\%,25\%, and $55 \%$, respectively. In our current study, we showed that the prevalence of clarithromycin resistant, clindamycin resistant, minocycline resistant, and ciprofloxacin resistant Streptococcus dysgalactiae subsp. equisimilis were $28 \%, 17 \%, 17 \%$, and $33 \%$, respectively. From those results, we suggested that the four antimicrobial resistant rate of Streptococcus dysgalactiae subsp. equisimilis were gradually decreasing. As macrolide is popular for treatment of respiratory infection disease in Japan [6] [9], the macrolide-resistant rate of Streptococcus pyogenes were increasing gradually [10]. Although both Streptococcus pyogenes and Streptococcus dysgalactiae subsp. equisimilis have beta-hemolytic activity, there were differences of the trend of antimicrobial susceptibility between Streptococcus dysgalactiae subsp. equisimilis and Streptococcus pyogenes. Further investigation about this point will be needed. Furthermore, we did not find any penicillin and cephalosporin-resistant Streptococcus dysgalactiae subsp. equisimilis in both our previous and current studies [8]. However, four incidents of penicillin-resistant Streptococcus dysgalactiae subsp. equisimilis isolated from blood cultures of three patients were reported in Denmark [12]. Thus, we need further antimicrobial surveil- 
lance to prevent the spread of penicillin-resistant Streptococcus dysgalactiae subsp. equisimilis. Our results suggest that the clinical and antimicrobial susceptible pattern are useful for screening the respiratory tract associated Streptococcus dysgalactiae subsp. equisimilius infection. Further continuous epidemiological analysis is needed for the clarification of several problems about respiratory tract associated Streptococcus dysgalactiae subsp. equisimilis.

\section{Acknowledgements}

We thank Mr. Masashi Ishihara and Ms. Miwako Fujimura for special encouragement. This study was supported by a grant-in-aid for research from the Nagoya City University, Japan.

\section{References}

[1] Facklam, R. (2002) What Happened to the Streptococci: Overview of Taxonomic and Nomenclature Changes. Clin Microb Rev, 15, 613-630. http://dx.doi.org/10.1128/CMR.15.4.613-630.2002

[2] Brandt, C.M. and Spellerberg, B. (2009) Human Infections Due to Streptococcus dysgalactiae Subspecies Equisimilis. Clin Infect Dis., 49, 766-772. http://dx.doi.org/10.1086/605085

[3] Vandamme, P., Pot, B., Falsen, E., Kersters, K. and Devriese, L.A. (1996) Taxaonomic Study of Lancefield Streptococcal Groups C, G, and L (Streptococcus dysgalactiae) and Proposal of S. dysgalactiae Subsp. Equisimilis Subsp. Nov. Int J Syst Bacteriol., 46, 774-781. http://dx.doi.org/10.1099/00207713-46-3-774

[4] Hashikawa, S., Iinuma, Y., Furushita, M., Ohkura, T., Nada, T., Torii, K., Hasegawa, T. and Ohta, M. (2004) Characterization of Group C and G Streptococcal Strains That Cause Streptococcal Toxic Shock Syndrome. J Clin Microbiol., 42, 186-192.

http://dx.doi.org/10.1128/JCM.42.1.186-192.2004

[5] Humar, D., Datta, V., Bast, D.J., Beall, B., De Azavedo, J.C.S. and Nizet, V. (2002) Streptoly$\sin S$ and Necrotizing Infections Produced by Group G Streptococcus. The Lancet, 359, 124129. http://dx.doi.org/10.1016/S0140-6736(02)07371-3

[6] Minami, M., Ichikawa, M., Matsui, H., Hata, N., Wakiyama, N., Matsumoto, M., Ohta, M. and Hasegawa, T. (2011) Prevalence of a Streptococcal Inhibitor of a Complement-Mediated Cell Lysis-Like Gene (sicG) in Streptococcus dysgalactiae Subsp. Equisimilis. Curr Microbiol., 62, 884-887. http://dx.doi.org/10.1007/s00284-010-9798-8

[7] Ichikawa, M., Minami, M., Ohashi, M., Wakimoto, Y., Matsui, H. and Hasegawa, T. (2011) Clinical and Microbiological Analysis of Beta Hemolytic Streptococci during 2006-2010 at Nagoya University Hospital. Nagoya Med J., 51, 175-189.

[8] Minami, M., Kuriyama, M., Ohshima, Y., Sakakibara, R., Imura, T., Morita, H., Kanemaki, N. and Ohta, M. (2015) Clinical Characteristics and Antimicrobial Susceptible Patterns of Streptococcus dysgalactiae Subsp. Equisimilius Isolates during 2009-2013 in Japan. Int. J. Curr. Microbiol. App. Sci., 4, 498-507.

[9] Takahashi, T., Sunaoshi, K., Sunakawa, K., Fujishima, S., Watanabe, H. and Ubukata, K., Invasive Streptococcal Disease Working Group (2010) Clinical Aspects of Invasive Infections with Streptococcus dysgalactiae Ssp. Equisimilis in Japan: Differences with Respect to Streptococcus pyogenes and Streptococcus agalactiae Infections. Clin Microbiol Infect., 16, 1097-1103. http://dx.doi.org/10.1111/j.1469-0691.2009.03047.x 
[10] Takahashi, T., Asami, R., Tanabe, K., Hirono, Y., Nozawa, Y., Chiba, N. and Ubukata, K. (2010) Clinical Aspects of Invasive Infection with Streptococcus dysgalactiae Subsp. Equisimilisin Elderly Patients. J Infect Chemother., 16, 68-71.

http://dx.doi.org/10.1007/s10156-009-0016-1

[11] Clinical and Laboratory Standards Institute (2014) Performance Standards for Antimicrobial Susceptibility Testing: 24st Information Supplement. Clinical and Laboratory Standards Institute M100-S24, Wayne.

[12] Fuursted, K., Stegger, M., Hoffmann, S., Lambertsen, L., Andersen, P.S., Deleuran, M. and Thomsen, M.K. (2016) Description and Characterization of a Penicillin-Resistant Streptococcus dysgalactiae Subsp. Equisimilis Clone Isolated from Blood in Three Epidemiologically Linked Patients. J Antimicrob Chemother. http://dx.doi.org/10.1093/jac/dkw320

Submit or recommend next manuscript to SCIRP and we will provide best service for you:

Accepting pre-submission inquiries through Email, Facebook, LinkedIn, Twitter, etc. A wide selection of journals (inclusive of 9 subjects, more than 200 journals)

Providing 24-hour high-quality service

User-friendly online submission system

Fair and swift peer-review system

Efficient typesetting and proofreading procedure

Display of the result of downloads and visits, as well as the number of cited articles

Maximum dissemination of your research work

Submit your manuscript at: http://papersubmission.scirp.org/

Or contact jbm@scirp.org 